\title{
Nivel óptimo de proteínas en la dieta para alevines de Prochilodus mariae
}

\author{
Optimal dietary protein levels for \\ Prochilodus mariae alevins
}

\begin{abstract}
The purpose of this work was to determine the optimal protein level to feed alevins of coporo (Prochidolus mariae) fish. Four diets were formulated with growing levels of crude protein (15, 25, 35 and 45\%) with similar energy values (between 19,6 and 20,4 KJ g-1). Three trials were performed for each diet. The animals were acclimated and fed a commercial diet during one week and after that time the test diets were administered ad libitum three times a day during 41 days. Fifty five frys per fishbowl were placed with an initial mean weight of 0,32 $\pm 0,04 \mathrm{~g}$. Three periods of fourteen days each were registered and at the end of each period weight gain (GP) and food consumption per fish tank was determined. The zootechnical parameters, final mean weights (PMF), total weight gain (GTP), specific growth rate (TCE) and daily growth index (ICD) of the fish did not show significant differences between the diets with protein levels of 35\% and $45 \%$ proteins, indicating that a diet with $35 \%$ protein is adequate for a good development of the fish in this stage.

Key words: Prochilodus mariae, coporo, alevins, diets, protein.
\end{abstract}

\section{INTRODUCCIÓN}

Desde la Segunda Guerra Mundial, la demanda de pescado para el consumo humano, ha ido en aumento, lo que ha fomentado una presión creciente sobre los recursos de la pesca. Como la demanda está acercándose a los límites de la producción, muchos recursos pesqueros están sufriendo deterioro, lo que ha llevado a una sobrepesca que tiene efectos devastadores sobre los ecosistemas (1).

La acuicultura, se ocupa del cultivo controlado de animales y plantas acuáticas en sus diferentes etapas que contemplan: cosecha, proceso, comercialización y consumo final. La piscicultura, encargada del cultivo de peces, es una de las ramas más importantes y más antiguas de la acuicultura (1).

Venezuela cuenta con una riqueza íctica de importancia, la cual no se conoce totalmente en lo que respecta a su biología básica como alimentación, crecimiento y reproducción, referencias que permitan su utilización en programas piscícola (2).

La producción de especies en cautiverio con potencialidad de cultivo, entre ellas el Prochilodus mariae (coporo), es de gran importancia; sin embargo, para obtener una producción exitosa de esta especie, es necesario establecer una alimenta-
Tomas Enrique Visbal B. (1)

Marielba Morillo S. (2)

Daisy Altuve P. (3)

Pierre Aguirre (4)

Ana Luisa Medina G. (3)

(1) Postgrado en Química de Medicamentos, Facultad de Farmacia y Bioanálisis, Universidad de Los Andes, Mérida Venezuela. (2) Postgrado Ciencias Médicas Fundamentales, Facultad de Medicina, Universidad de Los Andes, Mérida Venezuela.

3) Grupo Ecología y Nutrición, Departamento de Ciencias de los Alimentos. Facultad de Farmacia y Bioanálisis. Universidad de Los Andes, Mérida Venezuela. (4) Institute National de la Recherche Agronomique (INRA), Saint Pée sur Nivelle, Francia.

Dirigir la correspondencia a: Tomas E. Visbal B Facultad de Farmacia Universidad de Los Andes Sector Campo Oro, detrás del HULA Mérida 5101, Mérida, Venezuela Fono: 58-274-2403474 e-mail: tomasvisbal34@gmail.com

Este trabajo fue recibido el 9 de Abril de 2013 y aceptado para ser publicado el 22 de Mayo de 2013. ción adecuada, sobre todo en los primeros estadios de vida, ya que esta representa unos de los principales problemas en la producción de peces (3). Por ello, es importante conocer los requerimientos nutricionales de cada especie principalmente en nutrientes esenciales como las proteínas.

Existen pocos trabajos referentes a las necesidades nutricionales de alevines de coporo; establecer estas necesidades permitiría incrementar la densidad de siembra y mejorar la respuesta productiva, favoreciendo con esto la economía del país.

El coporo está dentro de las especies cultivadas en Venezuela, pero uno de los grandes inconvenientes, con los cuales se enfrentan los acuicultores, es la ausencia de dietas diseñadas para esta especie.

La producción mundial de harina de pescado es de siete millones de toneladas por año, donde la mayor parte de ésta, se usa para la acuicultura, la alimentación del ganado y en el sector avícola. Mientras esta demanda siga creciendo, se intensificará la competición con las especies productivas terrestres por unos recursos limitados, con consecuencias, tanto en los precios como en la disponibilidad (1).

La harina de pescado es la principal fuente de proteína 
para la preparación de alimentos destinados al consumo de los peces. Es rica en energía y en minerales, de alta digestibilidad y muy palatable para la mayoría de peces, contiene entre 60 y $80 \%$ de proteína cruda, de la cual el 80 al 95\% es digestible para los peces; se caracteriza por su alto contenido de lisina y metionina, los dos aminoácidos más limitantes en los alimentos de origen vegetal (4-6). Sin embargo, los elevados costos de la harina de pescado, ocasionados por la alta demanda del producto, han obligado a los productores a buscar nuevas alternativas de alimentación con dietas en las que se incluya muy poca cantidad de ésta, o en casos extremos a no utilizarla $(6,7)$.

Otro problema que se registra, es el alto nivel de proteína que se maneja en las dietas de la mayoría de las especies en los sistemas acuícolas (8), que en muchos casos no se corresponden a los requerimientos de las especies, lo que ocasiona un mayor costo y una alta contaminación de los cuerpos de agua por los desechos nitrogenados $(9,10)$.

El objetivo de este estudio, fue determinar el nivel óptimo de proteínas en las dietas para alevines de P. mariae (coporo) utilizando como principal fuente proteica la harina de pescado.

El logro de este objetivo permitirá a investigadores y piscicultores, formular dietas con materias primas alternativas, con un porcentaje de proteína adecuado para el mejor crecimiento del pez, que conllevará a la disminución de los costos de producción del alimento.

\section{MATERIALES Y MÉTODOS}

El presente trabajo fue realizado en el laboratorio de nutrición acuícola del Departamento de Ciencia de los Alimentos, Facultad de Farmacia y Bioanálisis de la Universidad de los Andes (ULA), Mérida Edo. Mérida, Venezuela, durante un periodo de 41 días.

Material biológico

Se utilizaron 660 alevines de coporo, que fueron obtenidos en la estación piscícola de la Universidad Nacional Experimental del Táchira (UNET), con un tiempo de vida de 35 días después de la eclosión. Fueron distribuidos en 12 peceras de vidrio ( $37 \times 28 \times 49 \mathrm{~cm}$; volumen: 50 L), colocándose 55 alevines por pecera (peso inicial promedio: 0,32 $\pm 0,04 \mathrm{~g}$ ). Durante el desarrollo de este trabajo, el manejo y tratamiento de los alevines se realizó cumpliendo con todas las normativas éticas exigidas internacionalmente.

Las 12 peceras de vidrio contaron con un sistema de recirculación de agua, teniendo entrada y salida individual para cada tanque, con un flujo continuo de 1,40 l/min, un sistema de filtro para la retención de impurezas y un filtro biológico para reducir las concentraciones de nitritos y nitratos.

Los parámetros físico-químicos del agua fueron controlados, la temperatura se mantuvo graduada con un termostato Lifetech Aquarium a $28,0 \pm 1^{\circ} \mathrm{C}$. Semanalmente se determinó la cantidad de nitritos y nitratos, manteniéndose las concentraciones $<0,02$ ppm, para ello se utilizó un Kit marca Aquarium pharmaceuticals (API). En la oxigenación se empleó un sistema de aireación permanente para mantener los niveles de oxígeno disuelto próximos a saturación y su medición se llevó a cabo con un oxímetro Sper Scientific.

\section{Preparación de las dietas}

Se ensayaron cuatro dietas experimentales: DPM15 (15\% PB); DPM25 (25\% PB); DPM35 (35\% PB) y DPM45 (45\% PB) (tabla 1). Se utilizó como agente ligante no nutritivo, carboximetilcelulosa (CMC). Las mezclas de vitaminas y minerales fueron elaboradas cumpliendo los requisitos de la NRC (11). Previo al suministro de las dietas a los peces, fueron trituradas y su granulometría ajustada a través de tamices a dos tamaños: $>0,3<0,5 y>0,5<1 \mathrm{~mm}$, comenzando la alimentación con el gránulo más pequeño. Las dietas fueron preservadas en envases herméticos, bajo refrigeración, hasta su posterior uso. Cada dieta se ensayó por triplicado $(n=3)$.

La alimentación de los peces se efectuó tres veces al día, hasta la saciedad aparente. Cada catorce días se pesaba la biomasa y se determinaba el consumo de cada tanque para los análisis posteriores, los peces muertos eran retirados y pesados, las heces y residuos de alimentos eran eliminados diariamente por sifoneo.

\section{Análisis químico}

Se realizaron análisis químico a las materias primas (tabla 2), dietas experimentales (tabla 3) y a las carcasas de los peces

\section{TABLA 1}

Formulación de las dietas experimentales con diferentes porcentajes de proteínas.

\begin{tabular}{|c|c|c|c|c|}
\hline Materia Prima \% & DPM15 & DPM25 & DPM35 & DPM45 \\
\hline Harina de pescado & 15 & 30 & 45 & 60 \\
\hline Almidón gelatinizado & 23 & 19 & 10 & 0 \\
\hline Harina de maíz amarillo & 28 & 28 & 20 & 16 \\
\hline Afrecho de trigo & 20 & 10 & 13 & 13 \\
\hline Aceite soya & 8 & 7 & 6 & 5 \\
\hline${ }^{1}$ Premix vitaminas & 1 & 1 & 1 & 1 \\
\hline${ }^{2}$ Premix minerales & 1 & 1 & 1 & 1 \\
\hline Ligante (CMC) & 4 & 4 & 4 & 4 \\
\hline Total & 100 & 100 & 100 & 100 \\
\hline
\end{tabular}

DPM15 (Dieta pescado maíz 15\% PB); DPM25 (Dieta pescado maíz 25\% PB); DPM35 (Dieta pescado maíz 35\% PB) y DPM45 (Dieta pescado maíz $45 \%$ PB).

${ }^{1}$ Premix de vitamina (vit.) (1\%), componentes por Kg: Cloruro de colina 200 g; vit. E 10 g; vit. C 20 g; Acetato de vit. A 500000 UI/g 1 g; vit. D3 a 100000 UI/g 2,5 g;

vit. B3 o PP (acido nicotínico) $1 \mathrm{~g}$; vit. B5 2g; vit B1 0,1 g; vit B2 0,4 g; vit B6 0,3 g; vit. B9 0,1 g; concentrado de vit. B12 $1 \mathrm{~g}$; vit. B8 $1 \mathrm{~g}$; meso inositol $30 \mathrm{~g}$; celulosa $729,6 \mathrm{~g}$.

2Premix de minerales, componentes por Kg: fosfato bicálcico $500 \mathrm{~g}$; carbonato de calcio $212 \mathrm{~g}$, cloruro de sodio 40 g; hidróxido de magnesio 124 g; sulfato de hierro 20 g;

sulfato de zinc $4 \mathrm{~g}$; sulfato de magnesio $3 \mathrm{~g}$; sulfato de cobre g; sulfato de cobalto0,02 g; ioduro de potasio 0,04 g; selenio de sodio 0,03 g, fluoruro de sodio $1 \mathrm{~g}$. (11). 
(inicio y final del ensayo) (tabla 5), de acuerdo a la metodología (12). El porcentaje de proteínas se calculó a partir del contenido de nitrógeno total de la muestra analizada $(\mathrm{N} \times 6,25)$ por el método Kjedahl, utilizando un dispositivo de auto-análisis Kjeltec 2300, después de someter la muestra a digestión en caliente con ácido sulfúrico concentrado en presencia de un catalizador. Para el análisis de los lípidos totales, se empleó el método de Soxhlet, para tal fin se utilizó el equipo VELP Soxhlet. La humedad se obtuvo por secado en estufa, para lo cual se utilizó una estufa MEMMERT a $103 \pm 1{ }^{\circ} \mathrm{C}$, durante 24 horas (hasta peso constante). La pérdida de agua en la muestra se calculó por diferencia de peso. El contenido de cenizas se determinó por incineración de las muestras en una mufla, marca Linberg Blue digital a $600^{\circ} \mathrm{C}$ hasta la obtención de cenizas blancas.

Parámetros zootécnicos y retenciones

Cada 15 días se pesaba el lote de alevines de cada pecera y se determinaba su consumo. Con los datos del peso medio inicial y final, peso corporal inicial y final de la biomasa, consumo total de alimentos, número y peso de los peces muertos, se calcularon los parámetros zootécnicos (13), tales como:

- Peso medio inicial: $(\mathrm{PMI})=$ Peso de la biomasa, inicial, en la pecera/ $n^{\circ}$ de peces en la pecera.

- $\quad$ Peso medio final $(P M F)=$ Peso de la biomasa, final, en la pecera/ $n^{\circ}$ de peces en la pecera.

- $\quad$ Ganancia de peso total: (GPT) = PCF-PCl.

- $\quad$ Ganancia de peso en \% de peso medio inicial: (GP\%PMI) =PMF-PMI x100/(PMI x (dias- 1$)$.

- $\quad$ Índice de consumo: $(I C)=(C O N S \% M S) / G P T$.

- $\quad$ Eficiencia alimenticia: $(E A)=$ Peso ganado (g)/alimento ingerido ( $\mathrm{g}$ ).

- $\quad$ Tasa de crecimiento especifica: (TCE)=log (PMF)- $\log (\mathrm{PMI})$ x100/(días-1).

- Índice de crecimiento diario: $($ ICD $)=100 \times((P M F) 1 / 3-$ (PMI) 1/3)/duración (días)).

- $\quad$ Coeficiente de eficiencia proteica: $(C E P)=$ Peso húmedo ganado/proteína cruda ingerida.

- $\quad$ Retención $=((\mathrm{PCF} \times$ composición final del pez $)-(\mathrm{PCI} \times$ composición inicial del pez))/(CONS $x$ composición del alimento) $\times 100$.

Análisis estadísticos

Todos los datos obtenidos, fueron sometidos a un análisis de varianza (ANOVA) simple, con test Student de Newman Keuls, utilizando el paquete estadístico Statgraphics Centurión $\mathrm{XVI}$, versión 16.1.17.

\section{RESULTADOS}

Análisis químico de las materias primas

La composición química de las materias primas utilizadas, como aporte proteico, en la formulación de las dietas, se presenta en la tabla 2.

Análisis químico de las dietas ensayadas

Los análisis químicos de las dietas suministradas a los alevines durante el ensayo se muestran en la tabla 3. Se puede observar los valores de \% de proteínas se ajustan a los valores teóricos propuestos, y las mismas son isolipidicas e isocalóricas.

\section{Parámetros zootécnicos}

Con los datos obtenidos se determinaron los parámetros zootécnicos (tabla 4), de los alevines de coporo, alimentados con dietas de diferentes concentraciones de proteínas, mostrando diferencias significativas entre datos, en función del alimento recibido $(p<0,05)$

TABLA 2

Análisis químico de las materias primas empleadas en la formulación de las dietas (\%).

\begin{tabular}{lcccc}
\hline Materia prima & Humedad & Proteínas & Lípidos & Cenizas \\
Harina Pescado & 6,99 & 68,00 & 7,88 & 15,50 \\
Harina de maíz amarillo & 10,39 & 8,37 & 1,94 & 0,66 \\
Afrecho de Trigo & 13,16 & 15,52 & 2,88 & 3,94 \\
\hline ENN: Extractos no nitrogenados = 100-(\%proteínas+\%lípidos+\%cenizas) & & &
\end{tabular}

ENN: Extractos no nitrogenados = 100-(\%proteínas+\%lípidos+\%cenizas)

TABLA 3

Composición química de las dietas.

\begin{tabular}{|c|c|c|c|c|}
\hline Análisis químico (\%) & DPM15 & DPM25 & DPM35 & DPM45 \\
\hline Materia Seca & 92,14 & 93,40 & 94,25 & 92,93 \\
\hline Proteínas & 15,63 & 26,00 & 35,98 & 45,42 \\
\hline Lípidos & 9,63 & 9,97 & 9,98 & 9,63 \\
\hline Cenizas & 4,41 & 6,41 & 9,04 & 11,01 \\
\hline ENN & 70,33 & 57,62 & 45 & 33,94 \\
\hline Energía Digestible(KJ g-1) & 19,6 & 20,0 & 20,2 & 20,4 \\
\hline
\end{tabular}

DPM15 (Dieta pescado maíz 15\% PB); DPM25 (Dieta pescado maíz 25\% PB); DPM35 (Dieta pescado maíz 35\% PB) y DPM45 (Dieta pescado maíz 45\% PB)

Energía Digestible $(E D)=$ Calculado según los valores teóricos (\%proteínas * 23,6) + (\%lípidos * 39,5) + (\% Carbohidratos * 17,2) $(14)$ 
Composición corporal de los peces

Los datos sobre el análisis químico de la carcasa de los peces (proteínas, lípidos, cenizas y humedad expresados en base seca) se muestran en la tabla 5 . Se realizaron al inicio del ensayo (tiempo 0) sobre una muestra de peces representativa y al final del experimento se analizaron la totalidad de peces de cada tanque, por cada tratamiento, (12). Los \% de proteínas, lípidos y cenizas mostraron diferencias significativas $(p<0,05)$ y el $\%$ de materia seca no presentó diferencias significativas $(p>0,05)$

Retención de proteína y lípidos en alevines de coporo, alimentados con dietas experimentales durante 41 días.

Al finalizar el ensayo, los peces fueron sacrificados y liofilizados, para la determinación de las retenciones de proteína y lípidos (12). Estos valores se expresaron en base seca y se presentan en la tabla 6 . Las retenciones de proteínas y lípidos no presentan diferencias significativas $(p>0,05)$.

\section{DISCUSIÓN}

La importancia de la determinación del óptimo proteico en dietas para alevines de coporo (P. mariae), radica en que permite tener una idea clara del \% de proteína necesaria para un mejor crecimiento, lo que se traduce en una disminución de costos de producción del alimento, además de una reducción significativa de los desechos nitrogenados, que se vierten a los afluentes, ya que el contenido de proteína en la dieta será el necesario para el pez.

Hay pocos estudios sobre requerimientos proteicos en alevines de coporo, sin embargo, según el aporte de Hernández et al. (2) sobre el efecto de la relación energía-proteína en larvas de coporo (Prochidolus mariae), dietas con $40 \%$ o más de proteínas y $2500 \mathrm{Kcal}$ Kg-1, lograron una mayor tasa de crecimiento.

Los requerimientos proteicos en alevines de varias especies de peces de cultivo han sido investigado por diferentes autores (15-20) demostrando que dichos requerimientos varían de una especie a otra, debido a los hábitos alimenticios, el tamaño y la temperatura del agua.

Una respuesta similar de requerimientos de proteínas en otra especie, fue la reportada, en juveniles de mexican silverside (Menidia estor), que con niveles proteicos entre 40 a $50 \%$ dieron los mejores resultados de crecimiento y supervivencia (15).

TABLA 4

Parámetros zootécnicos de los alevines de Prochilodus mariae (coporo) alimentados con las diferentes dietas experimentales durante 41 días $(X \pm D E)$.

\begin{tabular}{|c|c|c|c|c|}
\hline \multirow{2}{*}{$\begin{array}{l}\text { DATOS } \\
\text { ZOOTECNICOS }\end{array}$} & \multicolumn{4}{|c|}{ DIETAS } \\
\hline & DPM15 & DPM25 & DPM35 & DPM45 \\
\hline PMI (g) & $0,36 \pm 0,02 a$ & $0,35 \pm 0,01 a$ & $0,30 \pm 0,01 b$ & $0,27 \pm 0,02 c$ \\
\hline PMF (g) & $1,54 \pm 0,2 c$ & $2,06 \pm 0,1 b$ & $2,45 \pm 0,1 a$ & $2,56 \pm 0,3 a$ \\
\hline GTP (g) & $48,15 \pm 5,6 b$ & $77,16 \pm 16,2 a$ & $87,13 \pm 5,7 a$ & $98,53 \pm 13,5 a$ \\
\hline GP\% PMI & $327,73 \pm 75,3 a$ & $483,87 \pm 41,1 b$ & $712,62 \pm 60,9 c$ & $833,62 \pm 32,9 d$ \\
\hline IC & $3,93 \pm 0,4 b$ & $2,37 \pm 0,5 a$ & $1,89 \pm 0,2 a$ & $1,61 \pm 0,1 \mathrm{a}$ \\
\hline EA & $0,26 \pm 0,02 c$ & $0,43 \pm 0,1 a$ & $0,53 \pm 0,1 a, b$ & $0,62 \pm 0,1 b$ \\
\hline TCE (\%) & $3,52 \pm 0,4 c$ & $4,30 \pm 0,2 b$ & $5,11 \pm 0,2 a$ & $5,45 \pm 0,1 a$ \\
\hline ICD (\%) & $1,08 \pm 0,2 c$ & $1,38 \pm 0,1 b$ & $1,65 \pm 0,1 a$ & $1,75 \pm 0,1 a$ \\
\hline CEP(PER) & $1,51 \pm 0,1$ & $1,56 \pm 0,4$ & $1,40 \pm 0,2$ & $1,28 \pm 0,1$ \\
\hline
\end{tabular}

DPM15 (Dieta pescado maíz 15\% PB); DPM25 (Dieta pescado maíz 25\% PB); DPM35 (Dieta pescado maíz 35\% PB) y DPM45 (Dieta pescado maíz 45\% PB) $\mathrm{PMI}=$ Peso medio inicial; $\mathrm{PMF}=$ Peso medio final; $\mathrm{GTP}=$ Ganancia total de peso; $\mathrm{GP} \% \mathrm{PMI}=$ Ganancia total de peso en \% de peso inicial;

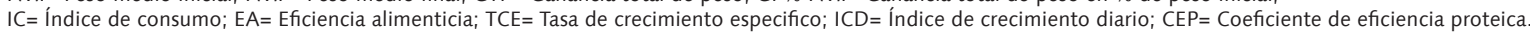
$a, b, c, d=$ Letras diferentes en las filas, indican diferencias significativas $(p<0,05)$.

\section{TABLA 5}

Análisis químico del de los alevines de Prochilodus mariae (coporo) alimentados con las diferentes dietas experimentales durante 41 días $(X \pm D E)$

\begin{tabular}{|c|c|c|c|c|c|}
\hline $\begin{array}{l}\text { Análisis químico } \\
(\%)\end{array}$ & $\begin{array}{c}\text { DIETAS } \\
\text { Lote Inicial }\end{array}$ & DPM15 & DPM25 & DPM35 & DPM45 \\
\hline Proteínas & $69,67 \pm 0,3$ & $47,02 \pm 1,1 c$ & $52,32 \pm 1,5 b$ & $58,30 \pm 1,9 a$ & $59,84 \pm 0,6 a$ \\
\hline Lípidos & $5,44 \pm 0,03$ & $35,91 \pm 1,5 a$ & $29,90 \pm 0,1 a$ & $19,66 \pm 0,3 b$ & $14,88 \pm 0,3 b$ \\
\hline Cenizas & $19,09 \pm 0,8$ & $13,70 \pm 0,4 c$ & $14,34 \pm 0,4 c$ & $16,04 \pm 0,8 b$ & $17,50 \pm 0,4 a$ \\
\hline Materia seca & $91,74 \pm 0,1$ & $94,83 \pm 0,4$ & $95,65 \pm 0,5$ & $95,09 \pm 0,6$ & $94,54 \pm 0,6$ \\
\hline
\end{tabular}

DPM15 (Dieta pescado maíz con 15\% PB); DPM25 (Dieta pescado maíz con 25\% PB); DPM35 (Dieta pescado maíz con 35\% PB) y DPM45 (Dieta pescado maíz con 45\% PB). Los datos se presentan corregidos por \% de materia seca.

$a, b, c, d=$ Letras diferentes, en las filas, indican diferencias significativas. $(p<0,05)$. 
Algunos parámetros de interés, empleados para determinar el crecimiento favorable en los peces son: peso medio final (PMF) y ganancia de peso total (GPT). El PMF de las dietas DPM35 (35\% PB) y DPM45 (45\% PB) tuvieron los mejores resultados, si los comparamos con las dietas DPM15 (15\% PB) y DPM25 (25\% PB).

En cuanto a la GTP, no se observaron diferencias entre DMP25, DPM35 y DPM45. Lo que nos indica que los \% proteínas más altos favorecen la ganancia de peso. Delmondes et al. (16), determinó que para alevines de curimbatá (Prochilodus affins), un 26,05 \% PB y $2700 \mathrm{Kcal} \mathrm{Kg}$-1, proporcionó una mejor repuesta en ganancia de peso y composición de la carcasas de los peces, siendo este \% proteico inferior al conseguido como óptimo en este trabajo.

En contraste, requerimiento de proteína superiores han sido reportados para otras especies, como juveniles de senegalese sole (Solea senegalensis), donde dietas con niveles proteicos comprendidos entre 53 y $59 \%$ presentaron mayor ganancia de peso (17). Otro estudio indicó que la mayor ganancia de peso, de crías del bagre balsas (Ictalurus balsanus), se obtuvieron utilizando dietas con $53 \%$ de proteína (18). Así, Kim et al. (19) indicaron que en alevines de olive flounder (Paralichthys olivaceus), dietas entre 50 y 55\% de proteínas produjeron una mayor ganancia de peso.

Para la determinación de la eficiencia de una dieta con respecto a otra, relacionando el contenido de sus nutrientes, se utiliza la tasa de crecimiento especifica (TCE), coeficiente de eficiencia proteica (CEP) y la ganancia de peso por $\%$ de peso medio inicial (GP\%PMI).

En cuanto a TCE existen diferencias entre las dietas, pero no hay diferencias entre la DPM35 y DPM45, lo que podría indicar que el óptimo de proteína se encuentra ubicado entre el 35 y $45 \%$ de proteína.

Mora et al. (20) no consiguieron diferencias significativas en la tasa de crecimiento, en alevines de bagre yaque (Leiarius marmoratus), alimentados con concentrado comerciales que contenían 28, 32 y 36\% de proteína bruta.

La GP\%PMI se encuentra entre 327,73 y 833,62; observando un aumento de este valor a medida que se incrementó el \%PB de la dieta, parámetro que mostró diferencias significativas entre las dietas.

Como se puede apreciar en la mayoría de los parámetros, a excepción del CEP (tabla 4), los valores aumentaron a medida que se incrementó el \% de proteína en las dietas. Igualmente, las dietas DPM35 y DPM45, fueron las que presentaron los mejores resultados y en gran parte de los datos zootécnicos no presentaron diferencias significativas entre ellas, pero si mostraron diferencias con las otras dos dietas.

En la tabla 5, se presenta la composición química de la carcasa de los peces (12), el valor de \%PB está entre 47,02 y 59,84, y mostró un incrementó directamente proporcional con el aumento del \%PB de la dieta, y el \% de lípidos en la carcasa de los peces, está entre 14,88 y 35,91 y mostró una disminución inversamente proporcional al incremento del \%PB de la dieta, que corresponde con los resultados esperados.

En cuanto a retención de proteína, no se observaron diferencias significativas (tabla 6), pero se observó que la misma se incrementó a medida que aumentaba el porcentaje de proteína, hasta la dieta DMP35, cuando comenzó a disminuir. Esto nos puede indicar que valores de \% PB mayores a 35\% no se traducen en un aumento de la retención proteica. En la retención de lípidos (tabla 6) los valores se ubicaron entre 90,52 y 92,18 y no se observaron diferencias significativas entre las dietas.

En el estudio realizado, el nivel óptimo de proteína en la dieta, se podría ubicar entre 35 y 45\% (DPM35 y DPM45), ya que estas proporcionaron un máximo crecimiento en alevines de coporo, basados en el peso medio final (PMF), ganancia total de peso (GTP) y tasa de crecimiento específico (TCE).

\section{CONCLUSIÓN}

Se puede concluir, según los parámetros zootécnicos, que una dieta con $35 \%$ de proteínas, podría ser la adecuada para obtener el máximo crecimiento de alevines de Prochilodus mariae (coporo), durante el tiempo que duró el ensayo. La formulación de la dieta a este \% de proteína reduciría el costo de alimentación de alevines de coporo.

\section{RESUMEN}

El propósito de este trabajo fue determinar el óptimo proteico en la alimentación de alevines de coporo (Prochidolus mariae). Se formularon 4 dietas con niveles crecientes de proteína cruda $(15,25,35$ y 45\%), y valores energéticos similares entre 19,6 y 20,4 KJ g-1, de manera que sean isocalóricas. Se realizaron tres repeticiones por cada dieta $(n=3)$. Los alevines fueron aclimatados y alimentados con una dieta comercial durante una semana y luego de este tiempo se le suministraron las dietas a ensayar ad libitum tres veces al día durante 41 días. Se colocaron 55 alevines por pecera con peso medio inicial de $0,32 \mathrm{~g} \pm 0,04$. Se llevó un registro durante tres períodos, de catorce días cada uno, al final de cada período se determinó la ganancia de peso (GP) y el consumo de alimento por pecera. Los parámetros zootécnicos, pesos medios finales (PMF), ganancia total de peso (GTP), tasa de crecimiento específico (TCE) e índice de crecimiento diario (ICD) de los peces, no presentaron diferencias significativas entre las dietas con 35 y $45 \%$ de proteínas, lo que indica que una dieta con $35 \%$ de proteínas, es adecuada para un buen

\section{TABLA 6}

Retenciones de proteínas y lípidos, de los alevines de Prochilodus mariae (coporo) alimentados con las diferentes dietas experimentales durante 41 días $(X \pm D E)$

$\begin{aligned} & \text { Retenciones (\%) } \text { DIETAS } \\ & \text { DPM15 }\end{aligned}$

Proteína

Lípidos

$$
\begin{gathered}
51,88 \pm 7,6 \\
91,85 \pm 17,4
\end{gathered}
$$

DPM25

$$
70,36 \pm 16,9
$$$$
91,39 \pm 4,5
$$

DPM35

$$
73,12 \pm 12,2
$$$$
92,18 \pm 2,0
$$

DPM45

$69,09 \pm 13,3$

$90,52 \pm 7,9$

DPM15 (Dieta pescado maíz 15\% PB); DPM25 (Dieta pescado maíz 25\% PB); DPM35 (Dieta pescado maíz 35\% PB) y DPM45 (Dieta pescado maíz 45\% PB).

Los valores indican que no hay diferencias significativas. $(p>0,05)$. 
desarrollo del pez en este estadio.

Palabras clave: Prochilodus mariae, coporo, alevines, dietas, proteína.

Agradecimientos: Al proyecto $\mathrm{CDCH}, \mathrm{FA}-438-08-03-$ ED "Elaboración de dietas, con sustitución parcial de la harina de pescado por materias primas no convencionales, para alevines de Prochilodus mariae (coporo). A la estación piscícola de la Universidad Nacional Experimental del Táchira (UNET), San Cristóbal Edo. Táchira, Venezuela, por haber suministrado los alevines de coporo para el ensayo. Al Laboratorio de Ciencia de los Alimentos de la Facultad de Farmacia y Bioanálisis de la Universidad de Los Andes, Mérida, Venezuela. AI INRA, Saint Pée Sur Nivelle, Francia, por su colaboración y asesorías.

\section{BIBLIOGRAFÍA}

1. Food and Agriculture Organization of the United Nations (FAO), The states of world fisheries and aquaculture. Roma, Italy. 2012.

2. Hernández G, González J, Alfonso E, Salmeron Y, Pizzani $P$. Efectos de la relación energía/proteína sobre el desempeño productivo en larvas de coporo (Prochidolus mariae). Zootecnia Trop. 2010; 28:173-82.

3. Patel A. Yakupitiyake A. Mexed feeding shedules in semiintensive pond cultura of Nile Tilapia, Oreochromis niloticus, $L_{;}$is it necessary to have two diets of differing protein contents, Aquaculture Res. 2003; 34:1343-52.

4. Lovell T. Practical feeding channel catfish. In: Lovell T. (Eds.) Nutrition and feeding of fish. Van Nostrand Reinhold, New York, EUA. 1989. p. 145-62.

5. Kikuchi K, Furuta T. Use of defatted soybean meal and blue mussel meat as substitute for fish meal in the diet of tiger puffer, Takifugu rubripes. J. World Aquaculture Soc. 2009; 40:472-82.

6. $\mathrm{Li} M H$, Peterson $C B$, Janes $C L$, Robinson EH. Comparison of diets containing various fish meal levels on growth performance, body composition, and insulin-like growth factor-I of juvenile channel catfish Ictalurus punctatus of different straits. Aquaculture 2006; 253:628-35.

7. Gaber MM. The effects of plant-protein-based diets supplemented with yucca on growth, digestibility, and chemical composition of nile tilapia (Oreochromis niloticus, L) fingerlings. J. World Aquaculture Soc. 2006; 37:74-82.

8. Abimorad E; Favero G; Castellani D; García F; Carneiro $D$. Dietary supplementation of lysine and/or methionine on performance, nitrogen retention and excretion in pacu
Piaractus mesopotamicus reared in cages. Aquaculture 2009; 295: 266-70.

9. Salhi M, Bessonart M, Chediak G, Bellagamba M, Carnevia D. Growth, feed utilization and body composition of black catfish, Rhamdia quelen, fry fed diets containing different protein and energy levels. Aquaculture 2004; 231:435-44.

10. Abdel-Tawwab M, Ahmad MH. Effect of dietary protein regime during the growing period on growth performance, feed utilization and whole-body chemical composition of nile tilapia, Oreochromis niloticus (L.), Aquaculture Res 2009; 40:1532-37.

11. National Research Conuncil (NCR). Nutrient requirements of warm water fishes and shellfishes. National Academy Press, Washington, D.C., 1993.

12. Association of Official Analytical Chemists. Official Methods of Analysis of the AOAC. 17th ed. Gaithersburg M. D, USA, 2000.

13. Guillaume J, Kaushik S, Bergot P, Metailler R. Nutrition et alimentation des poisons et crustacés, INRA/INFREMER. París, France. 1999.

14. Cho CY, Slinger SJ, Bayley SJ. Bioenergetics of salmonid fishes: energy intake, expenditure and productivity comp. Biochem Physiol. 1982; 73B:25-41.

15. Martínez C, Ríos M, Ambriz L, Jauncey K, Ross KJ. Dietary protein requirement of juvenile Mexican Silverside (Menidia estor Jordan 1879), a stomachless zooplanktophagous fish, Aquaculture Nutr. 2007; 13:304-10.

16. Delmondes $B M A$, Teixeira $L E A$, Serafini $M A$, Barbosa $R F$, Da Silva PK. Proteína bruta e energia digestível em dietas para alevinos de curimbatá (Prochilodus affins), R. Bras. Zootec 2005; 34:1795-806.

17. Rema P, Conceiçao L, Evers F, Castro-Cunha M, Dinis M, Dias J. Optimal dietary protein level in juvenile senegalese sole (Solea senegalensis). Aquaculture Nutr. 2008; 14:263-9.

18. Arce $E$, Luna J. Efecto de dietas con diferente contenido proteico en las tasas de crecimiento de crías del bagre del balsas Ictalurus balsanus (Pisces Ictaluridae) en condiciones de cautiverio. Rev. AquaTIC 2003; 18:39-47.

19. Kim KW, Wang XJ, Bal SC. Optimum dietary protein level for máximum growth of juvenile olive flounder Paralichthys olivaceus (Temminck et Schlegel). Aquaculture Res. 2002; 33:673-9.

20. Mora J, Moyetones F, Jover M. Influencia del contenido proteico en el crecimiento de alevines de bagre yaque, Leiarius marmoratus, alimentados con concentrados comerciales. Zootecnia Trop 2009; 27:187-94. 